NOTAS E INFORMAÇÕES / NOTES AND INFORMATIONS

\title{
A saúde bucal nas coortes de nascimentos de Pelotas, RS, Brasil
}

\section{The oral health studies in the Pelotas birth cohort studies, RS, Brazil}

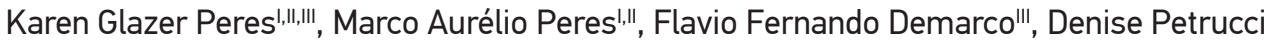
Gigante "II, Bernardo Lessa Horta"I", Ana Maria Baptista Menezes'"', Pedro Curi Hallal"', Alícia Matijasevich"', Iná Silva Santos"', Aluísio J D Barros"'I

\section{INTRODUÇÃO}

Três estudos prospectivos de base populacional com nascidos vivos foram realizados em 1982, 1993 e 2004 no município de Pelotas, RS. Os estudos de saúde bucal nas coortes de Pelotas foram planejados para investigar os principais agravos de saúde bucal que acometem os indivíduos em distintos períodos da vida, avaliar o padrão de comportamentos relacionados à saúde bucal, o papel das condições socioeconômicas ao longo da vida e seu impacto na saúde bucal, além de estimar a incidência de agravos bucais.

\section{QUEM FEZ PARTE DAS AMOSTRAS?}

$\mathrm{Na}$ coorte de 1982, foi obtida uma amostra sistemática com 70 (27\%) dos 259 setores censitários do município em 1997 ( $\mathrm{n}=1.076)$. Os estudos de saúde bucal partiram de uma subamostra aleatória $(\mathrm{n}=900)$ oriunda destes 1.076 entrevistados. Na coorte de 1993, uma subamostra de $13 \%$ das crianças acompanhadas do primeiro mês aos quatro anos de idade, incluindo todos os nascidos de baixo peso, foi obtida em 1998 ( $\mathrm{n}=1.273$ ). Os estudos de saúde bucal foram originados de uma subamostra destas crianças $(n=400)$. Na coorte de 2004 , o estudo de saúde bucal teve início em 2009 incluindo as crianças nascidas entre os meses de setembro e dezembro e que foram acompanhadas aos quatro anos de idade $(\mathrm{n}=1.303)$.

'Australian Research Centre for Population Oral Health, School of Dentistry, The University of Adelaide - Adelaide, Australia. "Programa de Pós-Graduação em Saúde Coletiva, Centro de Ciências da Saúde, Universidade Federal de Santa Catarina Florianópolis (SC), Brasil.

I'Programa de Pós-Graduação em Epidemiologia, Universidade Federal de Pelotas - Pelotas (RS), Brasil.

Autor correspondente: Karen Glazer Peres. Australian Research Centre for Population Oral Health, School of Dentistry, Faculty of Health Sciences. Level 1, 122 Frome Street, The University of Adelaide, SA 5005, Australia. E-mail: karen.peres@adelaide.edu.au Conflito de interesses: nada a declarar - Fonte de financiamento: Conselho Nacional de Desenvolvimento Científico e Tecnológico (CNPq), processos no 403362/2004-0, 476985/2004-5, 47081/2008-3, 402372/2008-5, 479621/2004-7 e 403257/2012-3. 


\section{QUANTOS ACOMPANHAMENTOS OCORRERAM?}

Na coorte de 1982, ocorreram estudos de saúde bucal aos 15 anos ( $\mathrm{n}=888,98,7 \%$ de participação), aos 24 anos $(\mathrm{n}=720)$ e aos 31 anos de idade $(\mathrm{n}=541)$. Na coorte de 1993 foi feito o acompanhamento das crianças aos 6 anos de idade $(\mathrm{n}=359)$ e aos 12 anos de idade ( $\mathrm{n}=339)$. Aos 15 anos de idade $(\mathrm{n}=339)$, informações autorreferidas de saúde bucal foram obidas, e, em 2012, um novo acompanhamento foi realizado aos 18 anos, expandindo a amostra para 1.203 indivíduos. Na coorte de 2004, o estudo de saúde bucal ocorreu aos cinco anos de idade $(\mathrm{n}=1.129)$.

\section{O QUE FOI INVESTIGADO?}

Os questionários aplicados face a face nos estudos de saúde bucal incluíram, no total, cerca de 140 variáveis. Os agravos bucais investigados foram: cárie dentária, problemas oclusais, condições periodontais, uso e necessidade de prótese, qualidade das restaurações em dentes posteriores, traumatismo dentário, fluorose, padrão de emergência dos molares permanentes, lesão em tecidos moles e presença de placa dentária ${ }^{1,2}$. Foram obtidas as aprovações pelo Comitê de Ética em Pesquisa da UFPel e o termo de consentimento dos participantes.

\section{QUAIS OS PRINCIPAIS RESULTADOS?}

A utilização de exames em dois quadrantes bucais diagonais foi uma medida efetiva para identificar fatores de risco para condições periodontais. Entretanto, pode subestimar a verdadeira prevalência dos desfechos, e sua validade depende da idade investigada ${ }^{3}$. Relatos maternos sobre higiene bucal de crianças com cinco anos de idade não foram bons substitutos para o exame de placa dental para avaliar o padrão de higiene ${ }^{4}$.

O índice CPO-D na coorte de 1982 apresentou um pequeno incremento dos 15 aos 24 anos de idade ${ }^{1}$, enquanto o CPO-D médio quase dobrou dos 12 (CPO-D = 1,2) aos 18 anos de idade (CPO-D = 2,1). Quase 70\% das crianças que apresentavam ceo-d igual a zero aos 6 anos permaneceram livres de cárie aos 12 anos de idade 5 , e cerca de dez anos mais tarde, em outra coorte, a média do índice (ceo-d) caiu quase para a metade. Houve diminuição significativa nas prevalências de mordida aberta anterior, mordida cruzada posterior e má oclusão de caninos entre 1993 e 2004 . A pobreza ao nascimento e episódios de pobreza do nascimento à vida adulta foram associados a um menor número de dentes saudáveis, e a ascensão econômica não reverteu os efeitos da pobreza ao nascimento sobre a saúde bucal ${ }^{6}$. Outros principais resultados são apresentados no Quadro 1. 
Quadro 1. Estudos de saúde bucal nas coortes de Pelotas, RS, em 1982, 1993 e 2004.

Perguntas de pesquisa

Existe associação entre obesidade e doença periodontal em adultos jovens?

Os problemas oclusais influenciam a satisfação com a aparência em adolescentes?

Fatores socioeconômicos influenciam a ocorrência de lesões bucais e a realização de autoexame em adultos?

Quais os determinantes do curso da vida na cárie dentária em crianças?

Quais os determinantes do curso da vida na qualidade de vida de adolescentes?

Qual o efeito da amamentação sobre os problemas oclusais na dentição decídua?

Quais os fatores de desenvolvimento infantil associados ao padrão de emergência dentária?

Quais os determinantes da dor dentária infantil?

Quais os fatores associados ao uso de serviço odontológico em crianças?
Principais resultados

Não, mas a inflamação sistêmica, o uso de fio dental e a frequência de escovação podem mediar a associação entre obesidade e gengivite.

Sim, as meninas com problemas oclusais mostraram-se mais insatisfeitas com sua aparência, independentemente de outros aspectos físicos presentes.

Adultos jovens de baixo nível socioeconômico tiveram maior prevalência de lesões bucais e realizam menos o autoexame.

Baixa escolaridade paterna, déficit na relação altura/idade aos 12 meses, não frequentar creche, doces e escovar os dentes < de 1 vez ao dia aos 6 anos.

Baixa escolaridade e desemprego materno, presença de cárie não tratada aos 6 e aos 12 anos, dor e apinhamento dentário e sangramento gengival.

Amamentação natural < do que 9 meses e uso de chupeta entre 12 meses e 1 ano de vida foram fatores de risco para mordida cruzada posterior.

Baixo peso ao nascer (dentes aos 6 e 12 meses), desnutrição aos 6 meses para número de dentes (12 meses) e para os primeiros molares aos 6 anos.

Baixa renda, mãe com $\leq 10$ dentes em alguma arcada e cárie não tratada.

Alta renda, escolaridade e o comportamento materno foram associados às visitas de rotina. Dor dentária e dentes com cárie associaram-se às visitas específicas.

\section{ASPECTOS FORTES E LIMITAÇÕES DOS ESTUDOS}

A alta taxa de resposta e a alta reprodutibilidade diagnóstica em todos os acompanhamentos, assim como o uso de instrumentos internacionalmente válidos ${ }^{1,2}$, destacaram-se nas pesquisas. A criação de projetos de extensão junto à Faculdade de Odontologia da UFPel com o objetivo de dar suporte às demandas de tratamento odontológicos oriundas das coortes serviu como 
estratégia para aumentar a taxa de resposta. Na coorte de 2004, passou-se a utilizar a unidade de superfície para estimativa do ataque de cárie em vez da unidade dente, permitindo aumentar a sensibilidade do índice frente à expressiva redução na gravidade da doença. Destaca-se como desafio para o futuro a possibilidade de acompanhamento dos mesmos indivíduos, a obtenção de financiamentos de longo prazo e a perspectiva de compreender a relação temporal ora dos agravos bucais como desfechos, ora como exposição para alguns agravos gerais, assim como a análise intergeracional dos membros das coortes.

\section{AGRADECIMENTOS}

Aos professores Cesar Gomes Victora e Fernando Celso Lopes de Barros, coordenadores das coortes, pelo apoio e por disponibilizar, sempre, os dados gerais das coortes.

\section{REFERÊNCIAS}

1. Peres KG, Peres MA, Demarco FF; Tarquínio SB, Gigante DP. Oral health studies in the 1982 Pelotas (Brazil) birth cohort: methodology and principal results at 15 and 24 years of age. Cad Saude Publica 2011; 27(8): 1569-80.

2. Peres MA, Barros AJD, Peres KG, Araújo CL, Menezes AM, Hallal PC et al. Oral health follow-up studies in the 1993 Pelotas (Brazil) birth cohort study: methodology and principal results. Cad Saude Publica 2010; 26(10): 1990-9.

3. Peres MA, Peres KG, Cascaes AM, Correa MB, Demarco FF, Hallal PC et al. Validity of partial protocols to assess the prevalence of periodontal outcomes and associated sociodemographic and behavior factors in adolescents and young adults. J Periodontol 2012; 83(3): 369-78.
4. Cascaes AM, Peres KG, Peres MA, Demarco FF, Santos IS, Matijasevich A, et al. Validade do padrão de higiene bucal em crianças aos 5 anos de idade relatado pelas mães. Rev Saúde Pública 2011; 45(4): 668-75

5. Peres MA, Barros AJD, Peres KG, Araújo CLP, Menezes ANB. Life course dental caries determinants and predictors in children aged 12 years: a populationbased birth cohort. Community Dent Oral Epidemiol 2009; 37(2): 123-33

6. Peres MA, Peres KG, Thomson WM, Broadbent JM, Gigante DP, Horta BL. The influence of family income trajectories from birth to adulthood on adult oral health: findings from the 1982 Pelotas birth cohort. Am J Public Health 2011; 101(4): 730-6. 